\title{
CDISC SDTM Collected Ethnicity Terminology
}

National Cancer Institute

\section{Source}

National Cancer Institute. CDISC SDTM Collected Ethnicity Terminology. NCI Thesaurus.

Code C128690.

Terminology associated with the ethnicity as collected codelist of the Clinical Data

Interchange Standards Consortium (CDISC) Study Data T abulation Model (SDT M). 